http://www.jfas.info

\title{
LORA PROPAGATION AT 433 MHZ IN TROPICAL CLIMATE ENVIRONMENT
}

\author{
K. A. Ahmad*, J. D. Segaran, F. R. Hashim and M. T. Jusoh
}

Faculty of Electrical and Electronic Engineering, National Defense University of Malaysia, Kuala Lumpur, Malaysia

Published online: 10 September 2017

\begin{abstract}
LoRa employs chirp spread spectrum technology and being considered as one of the promising system for Low-Power-Wide-Area-Network (LPWAN) to support the growth of Internet of Things (IoT) applications. Designed to operate in the industrial, scientific and medical (ISM) bands, LoRa had been tested and evaluated mainly in Europe and US in the $868 \mathrm{MHz}$ and $915 \mathrm{MHz}$ modulation bands. This paper provides some initial results in the performance of LoRa signal propagation of $433 \mathrm{MHz}$ modulation in tropical climate environments.
\end{abstract}

Keywords: LoRa; LPWAN;433 kHz; Propagation; Tropical climate.

Author Correspondence, e-mail: khairol@upnm.edu.my

doi: http://dx.doi.org/10.4314/jfas.v9i3s.31

\section{INTRODUCTION}

\subsection{Growth of IoT}

Internet of Things (IoT) is a fast growing communication model that enables connectivity for thousands of sensors with only one base station in creating a better application in our everyday life. Foresee ably, the sensor nodes will be equipped mostly with microcontrollers and transceivers for network communication and suitable protocol stacks which results in a 
better communication and networking system between users. IoT is expected to be the key point for the future growth in cellular networks. In its press release dated 3 Aug 2016,Machina Research estimated 27billionIoT connections by the year 2025 and $11 \%$ of them will use Low-Power-Wide-Area (LPWA) modules [1].Among the available LPWA technologies, LoRa is one of them that is being tested and evaluated for the wireless Low-Power-Wide-Area-Network (LPWAN) implementations.

\subsection{Previous Works}

LoRa technology was deployed by SemTech in 2012 as open source network and supported by LoRa Alliance. LoRa operates in unlicensed industrial, scientific and medical (ISM) radio bands using chirp spread spectrum innovation where each symbol is sent using a wide frequency band. The operating frequency used mostly in Europe is $868 \mathrm{MHz}$ and in the United States is $915 \mathrm{MHz}$ [2-3]. In Malaysia, Lora was tested using $433 \mathrm{MHz}$ where this frequency is under the unlicensed spectrum in the country. Several studies have been conducted on LoRa performance at $868 \mathrm{MHz}$ modulation [4-5].A comparison of LoRa in $868 \mathrm{MHz}$ and $433 \mathrm{MHz}$ was done by [6] in Europe.In urban environment, the IoT is aiming to develop smart cities with capabilities to provide a number of benefits in the management and optimization of traditional public services such as transport, parking system, lighting, tank level monitors, surveillance and so forth [7-8]. Other than urban application, LoRa-based network has also been deployed in maritime setting [4].However, not much work has been done up to this date on LoRa propagation performance in the tropical climate environments.

\subsection{Objective}

The main objective of this paper is to present the performance of LoRA modulation at 433 $\mathrm{MHz}$ in the tropical climate environment in Malaysia. From these initial results, further analysis may be pursued for deeper understanding of LoRa performance in the tropical environments.

\section{BASICS OF LORA TECHNOLOGY}

\subsection{LoRa Characteristics}

LoRa progresses under LPWAN technologies where this wireless communication system runs 
under a low power and long range of network, which makes it an interesting product for smart sensing technology in public infrastructures such as home automation, health wellbeing monitoring and smart metering. LoRa was designed to deploy end devices with limited energy consumption so that these end devices need not to transmit more than one byte at a time. Thus, this increases the battery lifetime of the devices up to 10 years[9]. In LoRa modulation, the spreading of the spectrum is achieved by addressing a chirp spread spectrum modulation which means it uses frequency chirps with variation of frequency over time to transmit signal with integrated forward error correction (FEC). The Semtech LoRa transceivers also secures bi-directional communication, both uplink and downlink transmission between transmitter and receiver. Among key properties of LoRa modulation include high robustness of the system, long range capabilities, low power consumption, bandwidth scalable, Doppler resistant and ranging or localization. Due to the high robustness of the system, i.e. its high bandwidth (BW) times symbol duration (T) or BWT product (which means BWT $>1$ ) where this spread spectrum takes more spectrum than they really need, LoRa signal will be highly resistant to signal interference. This device is both bandwidth and frequency scalable, for an instance, Semtech SX1272 has programmable bandwidth of $500 \mathrm{kHz}, 250 \mathrm{kHz}$ and $125 \mathrm{kHz}$. Since it is using the concept of chirp spread spectrum, this device operates under a wider bandwidth which results in low power consumption and demodulates $19.5 \mathrm{~dB}$ below the noise floor [10]. Doppler resistant occurs because of the mobility of a source of signal causing a small shift in the frequency of LoRa pulse.

\subsection{Key Parameters}

Several parameters that should be taken to account in LoRa modulation scheme are the bandwidth $(\mathrm{BW})$, spreading factor(SF), coding rate $(\mathrm{CR})$ and carrier frequency. Taking the Semtech SX1272 as an example, the carrier frequency or centre frequency is in the range of $860 \mathrm{MHz}$ and $1020 \mathrm{MHz}$ programmable in steps of $61 \mathrm{~Hz}$ [2]. Spreading factor is defined as the ratio between the symbol rate and the chip rate. The chip rate is equivalent to the bandwidth of the system. For example, if the transceiver uses a programmable bandwidth of $125 \mathrm{kHz}$, the chip rate shall be $125 \mathrm{kbps}$ and can be calculated as $2^{\mathrm{SF}}$. The higher the spreading factor, the higher is the signal to noise ratio and increasing the data packets and also time on air of the 
packet. In relation, higher spreading factors denote slower chirps. Bandwidth is the most important parameter of LoRa modulation since the chip rate depends on the bandwidth. The higher bandwidth could give higher data rate but lower sensitivity due to additional noise. Fig.1 below demonstrates LoRa modulation and explains how the bandwidth is closely related to the chirping rate. There is a series of upward chirps where the maximum band of frequency is reached and downward chirps where minimum frequency is reached. The discontinuity in the frequency described by the figure below shows that encoded information is being transmitted. Therefore, by doubling the bandwidth, the transmission rate also doubles.

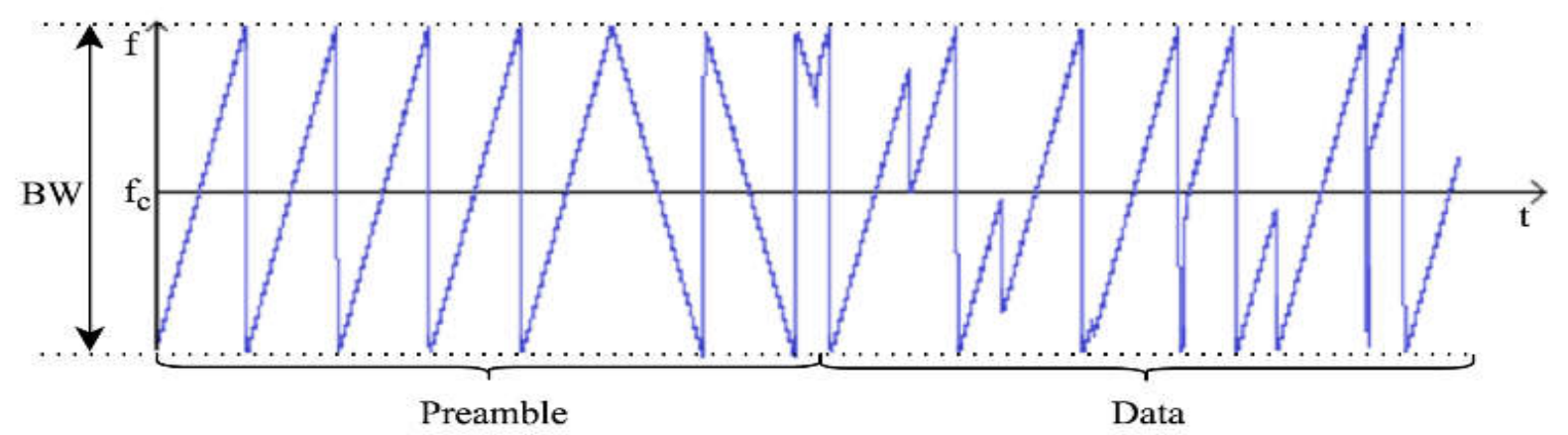

Fig.1.Frequency variation over time of signal transmitted[2]

Coding rate $(\mathrm{CR})$ is the forward error correction (FEC) mechanisms used by LoRa devices to protect against interferences. Increasing the CR will lower the Packet Error Rate in the presence of short burst of interferences. The coding rate is equivalent to $4 /(4+n)$ where $\mathrm{n}=0,1,2,3 \ldots \mathrm{CR}$ in the payload is located at the header of the packet [2].

\section{HARDWARES AND TEST SETUP}

In this work, RFM95W transceiver from HopeRF was used in both the transmitter and receiver modules. Internally, this unit integrates a SX1276 transceiver which enables the comparison between high and low frequencies. The wireless module was then mounted on a PCB which enables access to the module through serial port communications. The tests were set up outdoor in suburban/urban areas to observe its performance under line-of-sight (LOS) and also non line-of-sight (NLOS) in the tropical climate environment. In addition, the effects of varying the SF and BW on the RSSI were also tested. Omni directional antennas of $3 \mathrm{dBi}$ were used in the measurements. The transmit power was set at $23 \mathrm{dBm}$. Both transmitter and 
receiver modules were configured through a serial port communication, which also served as a monitor for both the transmitter and receiver. For the trials, a series of sequential data have been transmitted in a systematic manner. This not only allowed for the reception of data verification but also displaying the received signal strength indicator (RSSI) of each packet over the distance.As the RSSI indicates how well the receiver can hear signal from the access point, it is also useful for determining if the measured signal can get a good wireless connection. The higher the RSSI value, the better the signal is.

\section{RESULTS AND DISCUSSION}

This section presents the measurement results. First, it presents the measurement results of the LOS propagation test over outdoor environment around the campus of the National Defence University of Malaysia (NDUM). To this end, around 1000 packets were sent from the LoRa device to the receiver and the RSSIof received packets were recorded. All packets were sent with a bandwidth of $125 \mathrm{kHz}$, coding rate of $4 / 5$ and spreading factor of $128 \mathrm{chips} / \mathrm{symbol}$. In the second part, the parameters were configured to test on the effects of bandwidth and spreading factor on the reception. Finally, propagation effect in NLOS environment is to be presented.

\subsection{Line-of-Sight (LOS) Test}

In the LOS test, RSSI were measured for various ranges between the transmitter and the receiver. The transmitter was placed at ground level at coordinates $3.048577,101.722600$. The ranges were varied from $20 \mathrm{~m}$ to $1500 \mathrm{~m}$ under urban environment around NDUM (Fig. 2). However, as shown in Table 1, the minimum reported RSSI for successful communications was about $-98 \mathrm{dBm}$ under LOS at $1.17 \mathrm{~km}$ distance. This might suggest that the propagation distance of $433 \mathrm{MHz}$ LoRa signal in tropical environment is heavily attenuated. 


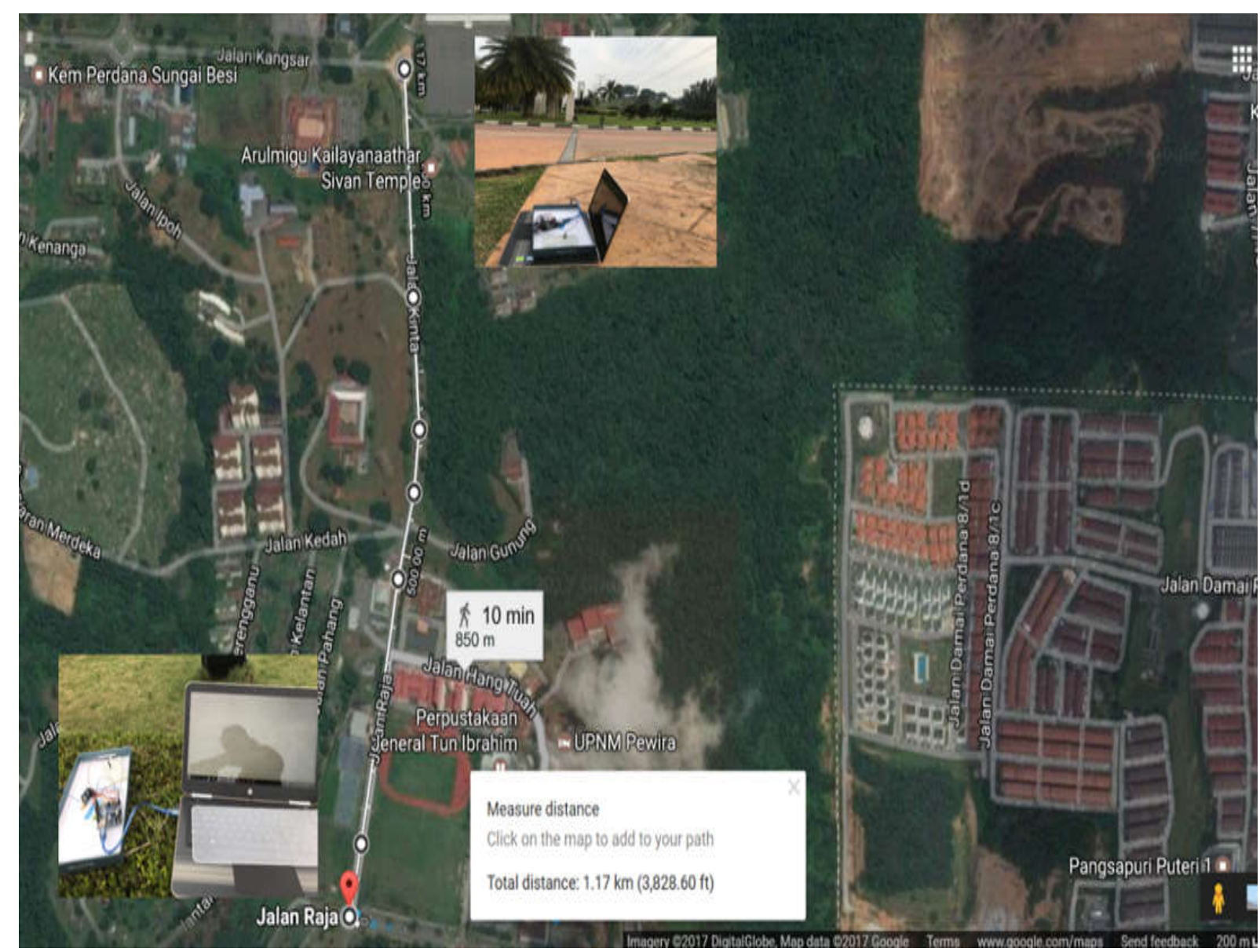

Fig.2.LOS test setup

Table 1. RSSI reading for LOS test

\begin{tabular}{cc}
\hline Distances(m) & RSSI (dBm) \\
\hline 20 & -80 \\
40 & -82 \\
60 & -89 \\
80 & -92 \\
100 & -86 \\
120 & -92 \\
140 & -90 \\
160 & -95 \\
180 & -93 \\
300 & -96 \\
1170 & -98 \\
\hline
\end{tabular}




\subsection{Effect of Spreading Factor (SF) and Bandwidth (BW) on the RSSI}

LoRa works on chirp spread spectrum modulation, where the most defining factor is the SF. Theoretically, the higher the SF, the longer the range it can propagate but with lower bitrate. To study the effect of LoRa's SF and BW in tropical climate, several measurements were taken for different SF and BW of LoRa. Fig. 3shows the test setup around Putrajaya. The transmitter was placed at high point to get a good LOS to the receiver. The separation between both of these devices is approximately $2 \mathrm{~km}$ and the elevation differences is about $80 \mathrm{~m}$. For this experiment, the LOS was sought out even though there is no perfect direct path.

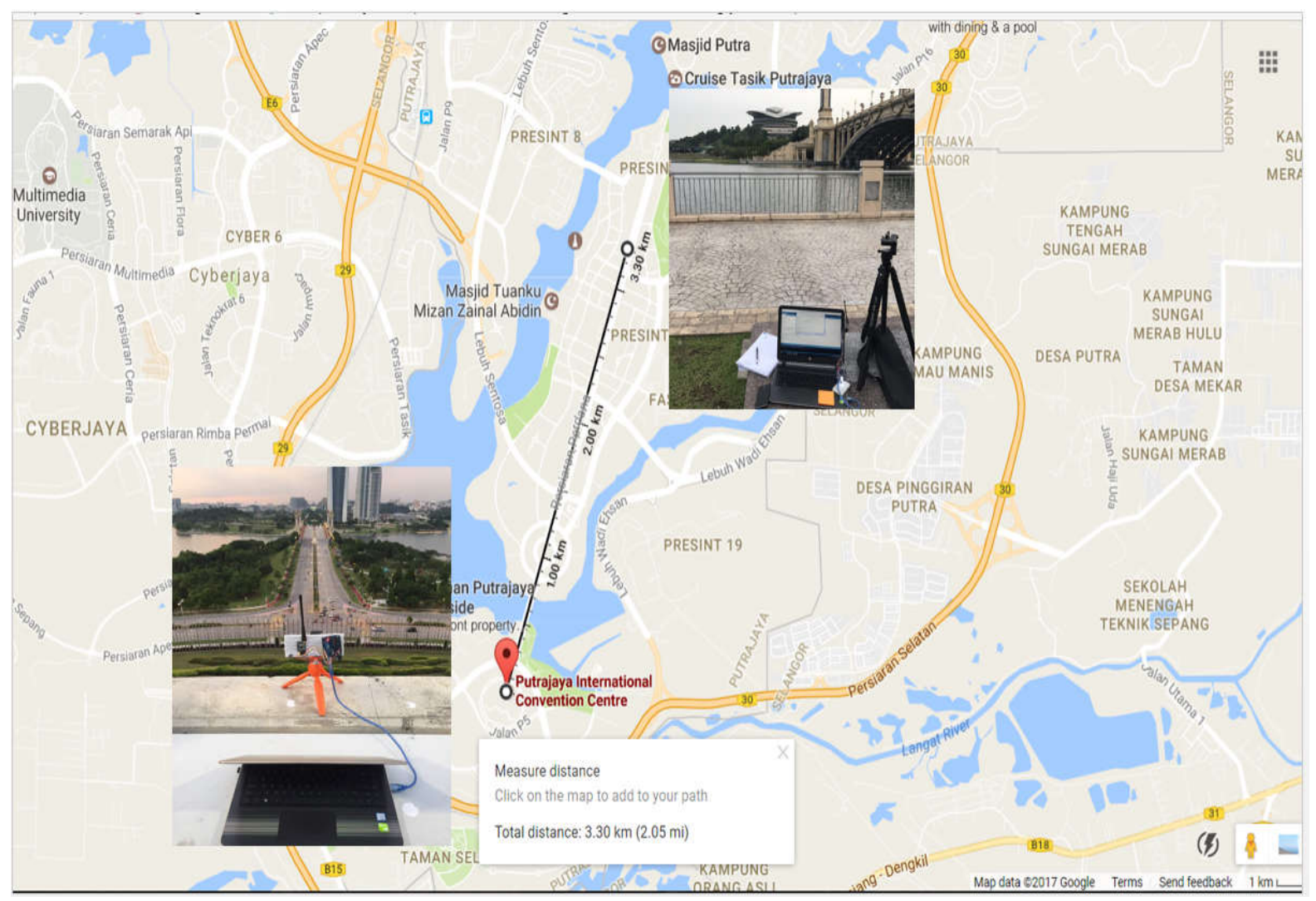

Fig.3.Test setup for SF and BW variations

In the measurements, the RSSI were extracted and as the packet data were being transmitted, some of them could not be received possibly due to the atmospheric attenuation. In Table 2, the bandwidth $500 \mathrm{kHz}$ with SF 128 chips/symbol gives a higher RSSI compared to the bandwidth $125 \mathrm{kHz}$ with the same SF. This indicates higher BW allows the signal to travel over longer range. As for bandwidth $31.25 \mathrm{kHz}$ with spreading factor of $512 \mathrm{chips} / \mathrm{symbol}$, the RSSI was the lowest even though the SF was higher the $125 \mathrm{kHz}$ and $500 \mathrm{kHz}$ BW. Lastly, for the same $125 \mathrm{kHz}$ bandwidth, the one with the higher SF had the higher RSSI. 
Table 2. RSSI reading for various SF and BW

\begin{tabular}{ccc}
\hline BW (kHz) & $\mathbf{2}^{\text {SF }}$ & RSSI (dBm) \\
\hline 31.25 & 512 & -101 \\
125 & 128 & -99 \\
125 & 4096 & -96 \\
500 & 128 & -90
\end{tabular}

With the information provided, it can be noticed that as the SF decreased, the RSSI value also decreased for a constant bandwidth. Since this technology operates in a wideband spread spectrum, higher spreading factor is applied in a LoRa modulation to achieve long range communication with higher data rates. Data is sent out as chip rate since it is equivalent to bandwidth. In order to evaluate the time on air, the symbol period, $\mathrm{T}_{\mathrm{s}}$ is defined as:

$T_{S}=\frac{2^{S F}}{B W}(1)$

As can be observed in Table 3, the symbol period (time on air) increased with increased SF. But when the bandwidth increased, the symbol period decreased since the bandwidth is wider and thus the time taken to transmit data is faster.

Table 3. RSSI reading for various SF and BW

\begin{tabular}{ccc}
\hline BW (kHz) & $\mathbf{2}^{\text {SF }}$ & Symbol Period, Ts (ms) \\
\hline 31.25 & 512 & 16.38 \\
125 & 128 & 1.02 \\
125 & 4096 & 32.77 \\
500 & 128 & 0.26 \\
\hline
\end{tabular}

\subsection{Non-Line-of-Sight (NLOS) Test}

Non-line-of-sight (NLOS) condition is established when the transmitted signal reached the receiver not by the direct path, but by other means such as reflection and diffraction. One of the features of LoRa is its ability to perform well in a multipath channel that the signal can still be received even after it passed through two or more walls of buildings. Besides, low frequencies provide great penetration of brick walls, trees and concretes so these frequencies usually have less loss than high frequency bands [11]. Hence, the NLOS test was setup in suburban environment where the signal strength where measured after it passed through a 
number of buildings. The test was conducted around residential area at Jalan Medan Bakri, Muar, Johor. The LoRa transceiver was tested with an output power of $+20 \mathrm{dBm}$. Table 4 shows the RSSI taken after being tested over 8 buildings. From the results, it can be observed that the signal was able to be received even after passing through a number of buildings which indicates its ability to propagate in NLOS condition where the degradation of the RSSI values was highly gradual.

Table 4. RSSI readings for NLOS test

\begin{tabular}{cc}
\hline No. of Building & RSSI (dBm) \\
\hline 1 & -96 \\
2 & -105 \\
3 & -105 \\
4 & -106 \\
5 & -107 \\
6 & -108 \\
7 & -109 \\
8 & -110 \\
\hline
\end{tabular}

\section{CONCLUSION}

From these initial results, there were indications that LoRa modulation of $433 \mathrm{kHz}$ in tropical climates suffered from some attenuation. Since LoRa uses chirp spread spectrum scheme, improvement in range could be achieved by manipulating the SF and BW of the signal but consideration must be given on their effects on the transmission bit rate. Nevertheless, LoRa's ability for its signal to propagate in NLOS condition was promising even in the tropical climate.

\section{ACKNOWLEDGEMENTS}

This research work is supported by the (UPNM/2016/GPJP/4TK/11) research grant. 


\section{REFERENCES}

[1] Machina Research. IoT global forecast and analysis 2015-2025.London: Machina Research, 2016

[2] Augustin A, Yi J, Clausen T, Townsley W M. A study of LoRa: Long range and low power networks for the Internet of Things. Sensors, 2016, 16(9):1-18

[3] Bor M, Vidler J, Roedig U. LoRa for the Internet of Things. In International Conference on Embedded Wireless Systems and Networks, 2016, pp. 361-366

[4] Petäjäjärvi J, Mikhaylov K, Pettissalo M, Janhunen J, Iinatti J. Performance of a low-power wide-area network based on LoRa technology: Doppler robustness, scalability, and coverage. International Journal of Distributed Sensor Networks, 2017, 13(3):1-16

[5] Aref M, Sikora A. Free space range measurements with SemtechLoRa ${ }^{\mathrm{TM}}$ technology. In2nd IEEE International Symposium on Wireless Systems within the Conferences on Intelligent Data Acquisition and Advanced Computing Systems: Technology and Applications, 2014, pp. 19-23

[6] Trasviña-Moreno C A, Blasco R, Casas R, Asensio Á. A network performance analysis of LoRa modulation for LPWAN sensor devices. InC. R.García, P. Caballero-Gil, M. Burmester, A. Quesada-Arencibia (Eds.),Ubiquitous Computing and Ambient Intelligence. UCAmI 2016, IWAAL 2016, AmIHEALTH 2016. Lecture Notes in Computer Science.Cham: Springer, 2016, pp. 174-181

[7] Zanella A, Bui N, Castellani A, Vangelista L, Zorzi M. Internet of things for smart cities. IEEE Internet of Things Journal, 2014, 1(1):22-32

[8] Cattani M, Boano CA, Römer K. An experimental evaluation of the reliability of LoRalong-range low-power wireless communication. Journal of Sensor and Actuator Networks, 2017, 6(2):2-8

[9] Semtech.Wireless and sensing products datasheet: SX1272/73-860MHz to $1020 \mathrm{MHz}$ low power long-range transceiver.California: SemtechCorporation, 2015

[10]Ducrot N, Ray D, Saadani A, Hersent O, Pop G, Remond G. LoRadevice developer guide. Technical Document, Rosh HaAyin: Orange, Connected Objects and Partnership, 2016 [11] Rizman ZI, Jusoff K, Rais SS, Bakar HH, Nair GK, Ho YK. Microwave signal 
propagation on oil palm trees: Measurements and analysis. International Journal on Smart Sensing andIntelligent Systems, 2011, 4(3):388-401

\section{How to cite this article:}

Ahmad K A, Segaran J D, Hashim F R, Jusoh M T. Lora propagation at $433 \mathrm{mhz}$ in tropical climate environment. J. Fundam. Appl. Sci., 2017, 9(3S), 384-394. 